\title{
DOXICICLINA: UMA REVISÃO SOBRE PARTICULARIDADES E UTILIZAÇÃO CLÍNICA NA ESPÉCIE EQUINA
}

\author{
D’EL REY-DANTAS, Fernanda Timbó ${ }^{1}$; \\ FEIJÓ, Lorena Soares ${ }^{1}$; \\ NOGUEIRA; Carlos Eduardo Wayne ${ }^{2}$; \\ CURCIO, Bruna da Rosa ${ }^{3}$.
}

Recebido: 05/01/2018

Aceito: 01/08/2018

${ }^{1}$ Pós-graduanda em Ciência Animal pelo PPGV/UFPEL; ${ }^{2}$ Professor titular no Departamento de Clínicas
Veterinárias/UFPEL; ${ }^{3}$ Professora adjunta no Departamento de Clínicas Veterinárias/UFPEL.

\section{RESUMO}

\begin{abstract}
A
$s$ tetraciclinas foram os primeiros antibacterianos de amplo espectro descritos, sendo uma das classes de antibacterianos mais utilizada na medicina veterinária. $\mathrm{O}$ uso em equinos não é tão expressivo, mas vem aumentando ao longo dos anos, especialmente por seu espectro de ação e baixo custo. $O$ objetivo do presente trabalho foi descrever as particularidades e aplicações da doxiciclina na prática clínica de equinos, bem como as perspectivas futuras de utilização deste fármaco. O levantamento de informações foi realizado através de pesquisa nas plataformas Pubmed e Mendeley. A doxiciclina é um antibacteriano bacteriostático utilizado principalmente em infecções causadas por microorganismos intracelulares, porém é eficaz também contra bactérias Gram-negativas e Grampositivas, clamídias, rickettsias, micoplasmas e alguns protozoários. Estudos recentes têm demonstrado outras propriedades desse fármaco além da antibacteriana, tais como ação anti-inflamatória e de inibição da lipase, colagenase, apoptose e angiogênese. Desta forma, a doxiciclina pode ser empregada como coadjuvante em diversas circunstâncias clínicas, com destaque para as afecções articulares. Os efeitos adversos são raros e estão relacionados ao desequilíbrio da microbiota intestinal. Mais estudos ainda são necessários acerca das propriedades não antibacterianas da doxiciclina e sua segurança, porém as perspectivas são promissoras.
\end{abstract}

Palavras-chave: Tetraciclinas. Metaloproteinases. Osteoartrite. Teratogenicidade. 


\section{INTRODUÇÃO}

As tetraciclinas foram os primeiros antibacterianos de amplo espectro descritos, tendo eficácia contra bactérias Gram-positivas, Gram-negativas, clamídias, micoplasmas, rickettsias e alguns protozoários (CHOPRA; ROBERTS, 2001). É a classe de antibacterianos mais utilizada na medicina veterinária, sendo rotineiramente empregada na produção animal. O uso em equinos e animais de companhia não é tão expressivo, mas vem aumentando ao longo dos anos, especialmente por seu espectro de ação e baixo custo (CASTILLO, 2013). Após a descoberta da primeira molécula dessa classe, a clortetraciclina, realizada por Benjamin Duggar em 1945, grandes esforços foram feitos para que novas tetraciclinas fossem obtidas. Deste modo, entre 1950 e 1970 ocorreu o desenvolvimento de diversos membros dessa classe de antimicrobianos, tanto na forma de produtos naturais quanto semissintéticos (PEREIRA-MAIA et al., 2010).

A doxiciclina foi desenvolvida em 1966, sendo uma molécula da segunda geração de tetraciclinas (CASTILLO, 2013). Dentro de sua classe, caracteriza-se por maior biodisponibilidade oral, maior caráter lipofílico e penetração tecidual, melhor atividade contra Gram-positivos e maior ligação a proteínas (AGWUH; MACGOWAN, 2006). As tetraciclinas são utilizadas em menor escala em equinos, quando comparado ao uso em animais de produção, mesmo sendo um fármaco de fácil acesso e com amplo potencial terapêutico (CASTILLO, 2013). O objetivo do presente trabalho foi descrever as particularidades e aplicações da doxiciclina na prática clínica de equinos, bem como as perspectivas futuras de utilização deste fármaco.

\section{METODOLOGIA}

Para se realizar o levantamento de informações sobre a utilização da doxiciclina na espécie equina, foram utilizados os termos "doxycycline" e "equine" nas plataformas de busca do Mendeley e do Pubmed, obtendo-se 46 e 73 artigos, respectivamente. A maior parte dos artigos estava disponível nas duas plataformas e todos os resumos foram lidos para avaliação da relevância. A exclusão dos artigos baseou-se na falta de correlação com o tema proposto e/ou ausência de achados relevantes para a revisão. Durante a elaboração desta revisão, esta busca foi repetida em diversas ocasiões. 


\section{MECANISMO DE AÇÃO E RESISTÊNCIA}

As tetraciclinas são antimicrobianos bacteriostáticos. Penetram na célula bacteriana através de transporte ativo ou passivo e se ligam de forma reversível à subunidade 30s do ribossomo bacteriano, impedindo que o tRNA associe-se ao ribossomo e promova a síntese proteica (SPEER et al., 1992). Moléculas mais lipofílicas são mais ativas que as hidrofílicas, por isso doxiciclina, minociclina e glicilciclina possuem maior atividade antimicrobiana que as demais tetraciclinas (CHOPRA; ROBERTS, 2001).

Com a disseminação e o uso indiscriminado das tetraciclinas no final dos anos 90, a resistência bacteriana contra os fármacos desta classe aumentou (PEREIRA-MAIA et al., 2010; SPEER et al., 1992). Existem dois mecanismos de resistência às tetraciclinas de maior relevância clínica: o efluxo de medicamento e a proteção ribossomal (PEREIRA-MAIA et al., 2010). No mecanismo por efluxo, proteínas transmembranares (Tet A) ejetam as tetraciclinas para fora da célula bacteriana, diminuindo seu nível intracelular. A proteção ribossomal é realizada por proteínas citoplasmáticas que impedem a ligação das tetraciclinas aos ribossomos (PEREIRA-MAIA et al., 2010; SPEER et al., 1992).

\section{ESPECTRO DE AÇÃO}

A utilização mais notória da doxiciclina em equinos é no tratamento da infecção por Anaplasma phagocytophilium (antiga Ehrliquia equi), agente etiológico da Erliquiose Granulocítica Equina. As tetraciclinas são as drogas de eleição no tratamento de erliquioses em diversas espécies, inclusive em humanos, sendo extremamente eficientes no controle destas bactérias intracelulares, apesar da pressão de seleção (DUMLER; BAKKER, 1995; NEER et al., 2002; PAPICH, 2003). Maurin et al. (2003) avaliaram a sensibilidade a diversos antimicrobianos de oito cepas de $A$. phagocytophilium isoladas de diferentes espécies animais, incluindo equinos. Todas as cepas foram suscetíveis a doxiciclina (concentração inibitória mínima $\leq 0,03 \mu \mathrm{g} / \mathrm{ml}$ ), sendo resistentes a ampicilina, amicacina, ceftriaxona, azitromicina e eritromicina.

A infecção causada por Neorickettsia risticii, que se caracteriza por causar diarreia aguda em equinos, é conhecida como Febre do Rio Potomac ou Erliquiose Monocítica Equina. Esta afecção também pode ser tratada com a doxiciclina (MAGDESIAN, 2015). É uma 
enfermidade sazonal que ocorre de forma endêmica na região sul do Rio Grande do Sul e Uruguai, especialmente nas proximidades da Lagoa Mirim. Apresenta alta letalidade, mas muitos animais se recuperam após o tratamento com tetraciclinas (COIMBRA et al., 2006; DUTRA et al., 2001).

Outros micro-organismos intracelulares que acometem os equinos, como a Lawsonia intracellularis, também são sensíveis a doxiciclina (SAMPIERI et al., 2006). Conforme já mencionado, este antibacteriano possui espectro de ação muito amplo, sendo eficaz contra diversos patógenos que causam infecções nesta espécie (CASTILLO, 2013).

Em estudo com 168 isolados bacterianos de equinos, Bryant et al. (2000) observaram sensibilidade significativa a doxiciclina (Tabela 1). Nos últimos anos, estudos com isolados clínicos de equinos tem demonstrado sensibilidade satisfatória a este antimicrobiano. Ferrer e Palomares (2018) analisaram dados retrospectivos de culturas e antibiogramas realizados a partir de swabs uterinos de éguas com metrite e observaram sensibilidade à doxiciclina em isolados Gram-negativos e Gram-positivos. Apenas o gênero Enterobacter spp. apresentou maior índice de resistência, com apenas $40 \%$ dos isolados demonstrando sensibilidade à doxiciclina. 
Tabela 1 - Concentração inibitória mínima de doxiciclina $(\mu \mathrm{g} / \mathrm{ml})$ necessária para inibir o crescimento em $50 \%$ $\left(\mathrm{CIM}_{50 \%}\right)$ e 90\% (CIM $\left.{ }_{90 \%}\right)$ em 168 isolados bacterianos de equinos enviados a um hospital veterinário.

\begin{tabular}{|c|c|c|c|c|}
\hline Micro-organismo & $\mathbf{N}$ & $\mathrm{CIM}_{50 \%}$ & $\mathrm{CIM}_{90 \%}$ & Variação \\
\hline \multicolumn{5}{|l|}{ Gram-negativos } \\
\hline Acinetobacter spp. & 6 & $\leq 0,12$ & $\leq 0,25$ & $\leq 0,6-2$ \\
\hline Aeromonas spp. & 1 & - & - & 0,25 \\
\hline Citrobacter freundii & 1 & - & - & $>4$ \\
\hline Enterobacter aerogenes & 8 & $>4$ & $>4$ & $1->4$ \\
\hline Enterobacter cloacae & 13 & $>4$ & $>4$ & $2->4$ \\
\hline Escherichia coli & 22 & $\leq 1$ & $>4$ & $0,5->4$ \\
\hline Flavobacterium spp. & 1 & - & - & 0,12 \\
\hline Gram-negative rod (unspeciated) & 4 & $\leq 0,12$ & $\leq 0,12$ & $0,12-1$ \\
\hline Klebsiella pneumoniae & 9 & $\leq 2$ & $>4$ & $0,12->4$ \\
\hline Klebsiella oxytoca & 2 & - & - & 1 \\
\hline Pasteurella haemolytica & 2 & - & - & $0,12-0,5$ \\
\hline Pasteurella pneumotropica & 6 & $\leq 0,25$ & $\leq 0,25$ & $0,12-1$ \\
\hline Pasteurella spp. & 7 & $\leq 0,25$ & $\leq 0,25$ & $0,12-0,5$ \\
\hline Proteus vulgaris & 1 & - & - & 2 \\
\hline Providencia rettgeri & 1 & - & - & $>4$ \\
\hline Pseudomonas aeruginosa & 13 & $>4$ & $>4$ & $>4$ \\
\hline Pseudomonas spp. & 5 & $\leq 0,12$ & $>4$ & $\leq 0,06->4$ \\
\hline Pseudomonas stutzeri & 1 & - & - & 1 \\
\hline Salmonella Group C1 & 4 & 2 & 2 & 2 \\
\hline Salmonella Group D & 2 & - & - & 2 \\
\hline Serratia marcescens & 6 & $>4$ & $>4$ & $2->4$ \\
\hline \multicolumn{5}{|l|}{ Gram-positivos } \\
\hline Rhodococcus equi & 5 & $\leq 0,25$ & $\leq 0,25$ & $\leq 0,06-2$ \\
\hline Staphylococcus aureus & 15 & $\leq 0,12$ & $\leq 0,25$ & $0,12->4$ \\
\hline Staphylococcus spp. & 3 & $\leq 0,06$ & $>4$ & $\leq 0,06->4$ \\
\hline Streptococcus Gr. D Enterococcus & 5 & $\leq 0,12$ & $>4$ & $0,12->4$ \\
\hline Streptococcus spp. & 2 & - & - & $\leq 0,06$ \\
\hline Streptococcus zooepidemicus & 20 & $\leq 0,06$ & $\leq 1$ & $\leq 0,06->4$ \\
\hline Streptococcus equi & 3 & $\leq 0,06$ & $\leq 0,12$ & $\leq 0,06-0,12$ \\
\hline
\end{tabular}

Fonte: Adaptado de BRYANT et al. (2000).

De forma semelhante, só que em estudo com isolados bacterianos de sinovite séptica, Robinson et al. (2016) avaliaram a sensibilidade bacteriana a diversos antibacterianos de primeira e segunda linha. A doxiciclina foi um dos mais eficientes in vitro, sendo eficaz contra a maior parte dos isolados Gram-positivos e negativos. 


\section{POSOLOGIA}

A doxiciclina está disponível em apresentações orais e injetáveis. Entretanto, em equinos, sua única indicação é a oral, visto que há relatos de colapso cardiovascular após a administração intravenosa (RIOND et al., 1992).

Em humanos, assim como em outras espécies, a alimentação não parece influenciar na absorção oral da doxiciclina, diferentemente do que acontece com as demais tetraciclinas (WELLING et al., 1977). Em equinos, no entanto, Davis et al. (2006) constataram melhor absorção da doxiciclina em jejum (12 horas), possivelmente pelo conteúdo fibroso da ingesta desta espécie, que formaria uma barreira física à absorção. Na rotina clínica é inviável a realização de jejum prévio às administrações de doxiciclina, visto que a posologia recomendada é $10 \mathrm{mg} / \mathrm{kg}$ a cada 12 horas. Entretanto, o ideal é que a alimentação seja evitada imediatamente antes e após a administração do medicamento, de forma que haja a mínima interferência possível na absorção (DAVIS et al., 2006).

Apesar da baixa biodisponibilidade relatada após administração oral em equinos (2,7\%), a doxiciclina atinge concentrações plasmáticas adequadas e é uma opção para o tratamento de infecções causadas por micro-organismos suscetíveis nesta espécie (DAVIS et al., 2006). Por ser altamente lipofílico, este fármaco tem a capacidade de se acumular no interior das células atingindo níveis intracelulares tão altos quanto os séricos. Em estudo realizado com equinos hígidos, Davis et al. (2006) observaram concentração máxima de doxiciclina 17 vezes mais alta em células polimorfonucleadas em relação ao plasma.

Em potros, a biodisponibilidade oral da doxiciclina parece ser superior, quando comparada a de equinos adultos (WOMBLE et al., 2007). A dose de $10 \mathrm{mg} / \mathrm{kg}$ a cada 12 horas resulta em concentrações plasmáticas mais altas que em adultos, além de níveis pulmonares considerados eficientes contra Rhodococcus equi e Streptococcus beta-hemolíticos, as quais são as principais causas de pneumonia em potros até 6 meses de idade (WOMBLE et al., 2007). Entretanto, apesar de atingir níveis plasmáticos que seriam eficazes, em estudo com a utilização de culturas de macrófagos de equinos infectados experimentalmente, a doxiciclina não se mostrou eficaz contra o Rhodococcus equi (GIGUÈRE et al., 2015). 


\section{UTILIZAÇÃO TERAPÊUTICA}

Além da atividade antibacteriana, outras propriedades das tetraciclinas vêm sendo estudadas. Em menor ou maior grau, a depender do efeito estudado, as moléculas desta classe apresentam ação anti-inflamatória e de inibição da lipase, colagenase, apoptose e angiogênese, além de outras funções (SAPADIN; FLEISHMAJER, 2006).

Diversos estudos (BRYANT et al., 2000; FORTIER, 2009; MAHER et al., 2014; SCHNABEL et al., 2010; WANG et al., 2017) vêm sendo realizados avaliando outras aplicações da doxiciclina, notadamente em doenças degenerativas. Mais recentemente, Wang et al. (2017) evidenciaram possíveis efeitos benéficos de baixas doses de doxiciclina no metabolismo da glicose em modelo experimental animal de diabetes. Neste estudo foi observada diminuição da inflamação sistêmica e melhora no controle glicêmico, perfil lipídico, além de morfologia e função de ilhotas pancreáticas, abrindo novas perspectivas para a utilização deste fármaco.

Em equinos, os efeitos não-antimicrobianos da doxiciclina têm sido pesquisados desde o início dos anos 2000. A aplicação mais promissora é como adjuvante no tratamento da osteoartrite, visto que a administração oral promove altas concentrações intra-articulares (BRYANT et al., 2000; SCHNABEL et al., 2010). Estudos recentes, com doses mais baixas (5 $\mathrm{mg} / \mathrm{kg}$ ) que as preconizadas para tratamento de infecções, demonstraram, além do acúmulo intra-articular do fármaco, inibição de metaloproteinases no líquido sinovial, o que seria desejável no tratamento das osteoartrites (MAHER et al., 2014). É importante ressaltar ainda que esses estudos sugerem que não há risco de indução de resistência bacteriana nessa posologia, já que as concentrações de doxiciclina no plasma e no líquido sinovial permanecem abaixo do $\mathrm{CIM}_{50 \%}$ da maior parte dos micro-organismos (MAHER et al., 2014; SCHNABEL et al., 2010). Essa é uma observação particularmente importante no contexto atual de emergência de bactérias super-resistentes e é mais um fator encorajador de pesquisas na área.

Atualmente a recomendação de baixas doses de doxiciclina como tratamento adjuvante da osteoartrite tem sido realizada da seguinte forma: durante a primeira semana de tratamento, administra-se $5 \mathrm{mg} / \mathrm{kg}$ uma vez por dia. Na segunda semana, utiliza-se $5 \mathrm{mg} / \mathrm{kg}$ a cada 48 h e, na terceira semana, esta mesma dosagem a cada 72 h (FORTIER, 2009). Uma 
observação importante é que as tetraciclinas possuem alta ligação proteica (DAVIS et al., 2006) e, portanto, não devem ser utilizadas concomitantemente a outras drogas com essa característica, como a fenilbutazona, por exemplo.

\section{EFEITOS ADVERSOS}

Em geral, a doxiciclina e outras tetraciclinas são drogas seguras (CASTILLO, 2013). Assim como outros antimicrobianos, o principal efeito adverso que pode ser observado com seu uso em equinos é a diarreia causada pela alteração da microbiota intestinal. Esse efeito, no entanto, é pouco frequente. Com a utilização da dose terapêutica de doxiciclina $(10 \mathrm{mg} / \mathrm{kg})$, apenas Barr et al. (2013) relataram incidência de diarreia. Mesmo assim, de 453 animais que receberam o fármaco, apenas quatro desenvolveram esse tipo de alteração.

Em humanos, o uso das tetraciclinas não é recomendado durante a gestação e em indivíduos em fase de crescimento devido o risco de deposição em ossos e dentes durante a calcificação (PEREIRA-MAIA et al., 2010). Entretanto, é questionável se isso seria válido para todos os fármacos dessa classe. Cross et al. (2016) realizaram uma revisão sistemática avaliando o uso de doxiciclina em mulheres gestantes e crianças e observaram que não há correlação entre a utilização do antibacteriano e efeitos teratogênicos ou descoloramento dos dentes em crianças. Isso sugere que, diferentemente da tetraciclina, a doxiciclina é um antibiótico de uso seguro nestas categorias.

Em equinos, existem poucos estudos avaliando o uso de antibacterianos durante a gestação (MACPHERSON et al., 2012; MACPHERSON et al., 2017; MURCHIE et al., 2006; SANTSCHI; PAPICH, 2000). Com relação à doxiciclina, bem como qualquer outra tetraciclina, ainda não foram publicados resultados em éguas gestantes. Dados preliminares de estudos conduzidos pelo ClinEq (Grupo de Ensino, Pesquisa e Extensão em Clínica Médica de Equinos/UFPEL) demonstraram não haver qualquer prejuízo a curto prazo para o potro na utilização da doxiciclina a partir dos 300 dias de gestação em dose única e a partir dos 320 dias na posologia recomendada (dados não publicados). Entretanto, mais avaliações ainda são necessárias para atestar a segurança deste fármaco nestas circunstâncias. 


\section{CONCLUSÃO}

A doxiciclina é um antibacteriano com amplas possibilidades de aplicação na clínica de equinos, porém muitas vezes subutilizado. Além do amplo espectro de ação contra microorganismos que comumente infectam esta espécie, sua utilização como anti-inflamatório e adjuvante no tratamento da osteoartrite apresentam grande potencial na medicina equina. Ademais, a perspectiva de utilização com segurança durante a gestação representa uma possibilidade promissora para a doxiciclina na espécie equina. Mais estudos são necessários visando melhor esclarecer suas propriedades não-antibacterianas e segurança na utilização nessas situações.

\section{DOXYCYCLINE: A REVIEW REGARDING PROPERTIES AND CLINICAL USE IN THE EQUINE SPECIES}

\section{ABSTRACT}

$\mathrm{T}$ etracyclines were the first broad spectrum antibacterials described, being one of the most widely used classes of antibacterials in veterinary medicine. The use in the equine species is not as expressive, but it is increasing over the years, especially for its broad spectrum and low cost. The aim of the present study was to describe the particularities and applications of doxycycline in the clinical practice of horses, as well as the future prospects of usage. The information was collected using Pubmed and Mendeley platforms. Doxycycline is a bacteriostatic antibacterial used mainly in infections caused by intracellular microorganisms, but its spectrum of action goes far beyond, being effective against Gram-negative and positive bacteria, chlamydia, rickettsia, mycoplasma and some protozoa. Recent studies have demonstrated other properties besides antibacterial, such as anti-inflammatory action and inhibition of lipase, collagenase, apoptosis and angiogenesis. In this way, doxycycline can be used as a coadjuvant in several clinical circumstances, especially in articular affections. Adverse effects are rare and related to intestinal microbiota imbalance. Further studies are still needed about the non-antibacterial properties of doxycycline and its safety, but the prospects are promising.

Keywords: Tetracyclines. Metalloproteinases. Osteoarthritis. Teratogenicity. 


\section{DOXICICLINA: UNA REVISIÓN SOBRE PARTICULARIDADES Y UTILIZACIÓN CLÍNICA EN LA ESPECIE EQUINA}

\section{RESUMEN}

as tetraciclinas fueron los primeros antibacterianos de amplio espectro descritos, siendo una de las clases de antibacterianos más utilizada en la medicina veterinaria. El uso en equinos no es tan expresivo, pero viene aumentando a lo largo de los años, especialmente por su espectro de acción y bajo costo. El objetivo del presente trabajo fue describir las particularidades y aplicaciones de la doxiciclina en la práctica clínica de equinos, así como las perspectivas futuras de utilización de este fármaco. El levantamiento de informaciones fue realizado a través de investigación en las plataformas Pubmed y Mendeley. La doxiciclina es un antibacteriano bacteriostático utilizado principalmente en las infecciones causadas por microorganismos intracelulares, pero su espectro de acción va mucho más allá, siendo eficaz contra bacterias Gram-negativas y positivas, clamidias, rickettsias, micoplasmas y algunos protozoarios. Los estudios recientes han demostrado otras propiedades de este fármaco además de la antibacteriana, tales como la acción antiinflamatoria y la inhibición de la lipasa, la colagenasa, la apoptosis y la angiogénesis. De esta forma, la doxiciclina puede ser empleada como coadyuvante en diversas circunstancias clínicas, con destaque para las afecciones articulares. Los efectos adversos son raros y están relacionados con el desequilibrio de la microbiota intestinal. Más estudios todavía son necesarios acerca de las propiedades no antibacterianas de la doxiciclina y su seguridad, pero las perspectivas son prometedoras.

Palabras clave: Tetraciclinas. Metaloproteinases. Osteoartritis. Teratogenicidad.

\section{REFERÊNCIAS}

AGWUH, K. N.; MACGOWAN, A. Pharmacokinetics and pharmacodynamics of the tetracyclines including glycylcyclines. Antimicrobial Agents and Chemotherapy, v. 58, p. 256-265, 2006.

BARR, B. S.; WALDRIDGE, B. M.; MORRESEY, P. R.; et al. Antimicrobial-associated diarrhoea in three equine referral practices. Equine Veterinary Journal, v. 45, p. 154-158, 2013.

BRYANT, J. E.; BROWN, M. P.; GRONWALL, R. R.; et al. Study of intragastric administration of doxycycline: pharmacokinetics including body fluid, endometrial and minimum inhibitory concentrations. Equine Veterinary Journal, v. 32, n. 3, 2000.

CASTILLO, J. R. E. Tetracyclines. In: GIGUÈRE, S.; PRESCOTT, J. F.; DOWLING, P. M. Antimicrobial therapy in veterinary medicine. Hoboken: WileyBlackwell, 2013. Cap. 15, p. 257-268. 
CHOPRA, I.; ROBERTS, M. Tetracycline antibiotics: mode of action, applications, molecular biology, and epidemiology of bacterial resistance. Microbiology and Molecular Biology Reviews, v. 65, n. 2, p. 232-260, 2001.

COIMBRA, H. S.; FERNANDES, C. G.; SOARES, M. P.; et al. Ehrliquiose monocítica equina no Rio Grande do Sul: aspectos clínicos, anátomo-patológicos e epidemiológicos. Pesquisa Veterinária Brasileira, v. 26, n. 2, p. 97-101, 2006.

CROSS, R.; LING, C.; DAY, N. P. J.; et al. Revisiting doxycycline in pregnancy and early childhood - time to rebuild its reputation? Expert opinion on drug safety, v. 15, n. 3, p. 367382, 2016.

DAVIS, J. L; SALMON, J. H.; PAPICH, M. G. Pharmacokinetics and tissue distribution of doxycycline after oral administration of single and multiple dosis in horses. American Journal of Veterinary Research, v. 67, n. 2, p. 310-316, 2006.

DUMLER, J. S.; BAKKEN, J. S. Ehrlichial diseases of humans: emerging tick-borne infections. Clinical Infectious Diseases, v. 20, p. 1102-1110, 1995.

DUTRA, F.; SCHUCH, L. F.; CURCIO, B. R.; et al. Equine monocyte ehrlichiosis in Uruguay and southern Brazil. Journal Veterinary Diagnostic investigation, v. 13, p. 433-437, 2001.

FERRER, M. S.; PALOMARES, R. Aerobic uterine isolates and antimicrobial susceptibility in mares with post-partum metritis. Equine Veterinary Journal, v. 50, n. 2, p. 202-207, 2018.

FORTIER, L. A. Current concepts in joint therapy. In: INTERNATIONAL CONGRESS OF WORLD EQUINE VETERINARY ASSOCIATION, 11, 2009, Guarujá. PROCEEDINGS. São Paulo: WEVA, 2009.

GIGUÈRE, S.; BERGHAUS, L. J.; LEE, E. A. Activity of 10 antimicrobial agents against intracellular Rhodococcus equi. Veterinary Microbiology, v. 178, n. 3-4, p. 275-278, 2015.

MACPHERSON, M. L.; GIGUERE, S.; HATZEL, J. N.; et al. Disposition of desfuroylceftiofur acetamide in serum, placental tissue, fetal fluids, and fetal tissues after administration of ceftiofur crystalline free acid (CCFA) to pony mares with placentitis. Journal of Veterinary Pharmacology and Therapeutics, v. 36, n. 1, p. 59-67, 2012.

MACPHERSON, M. L.; GIGUERE, S.; POZOR, M. A.; et al. Pharmacokinetics of ceftiofur sodium in equine pregnancy. Journal of Veterinary Pharmacology and Therapeutics, v. 40, n. 6, p. 17, 2017.

MAGDESIAN, K. G. Update on antimicrobial selection and use. In: SPRAYBERRY, K. A.; ROBINSON, N. E. Robinson's current therapy in equine medicine. St. Louis, MO: Elsevier Saunders, 2015. Cap. 33, p. 144-149. 
MAHER, M. C.; SCHNABEL, L. V.; CROSS, J. A.; et al. Plasma and synovial fluid concentration of doxycycline following low-dose, low-frequency administration, and resultant inhibition of matrix metalloproteinase-13 from interleukin-stimulated equine synoviocytes. Equine Veterinary Journal, v. 46, p. 198-202, 2014.

MAURIN, M.; BAKKEN, J. S.; DUMLER, J. S. Antibiotic susceptibilities of Anaplasma (Ehrlichia) phagocytophilumstrains from various geographic areas in the United States. Antimicrobial Agents and Chemotherapy, v. 47, n. 1, p. 413-415, 2003.

MURCHIE, T. A.; MACPHERSON, M. L.; LEBLANC, M. M.; et al. Continuous monitoring of penicillin $\mathrm{G}$ and gentamicin in allantoic fluid of pregnant pony mares by in vivo microdialysis. Equine Veterinary Journal, v. 38, n. 6, p. 520-525, 2006.

NEER, T. M.; BREIDTSCHWERDT, E. B.; GREENE, R. T; LAPPIN, M. R. Consensus statement on ehrlichial disease of small animals from the Infectious Diseases Study Group of the ACVIM. Journal of Veterinary Internal Medicine, v. 16, p. 309-315, 2002.

PAPICH, M. G. Antimicrobial therapy for gastrointestinal disease. The Veterinary Clinics of North America Equine Practice, v. 19, p. 645-663, 2003.

PEREIRA-MAIA, E. C.; SILVA, P. P.; ALMEIDA, W. B.; et al. Tetraciclinas e glicilciclinas: uma visão geral. Química Nova, v. 33, n. 3, p. 700-706, 2010.

RIOND, J. L.; RIVIERE, J. E.; DUCKETT, W. M.; et al. Cardiovascular effects and fatalities associated with intravenous administration of doxycycline to horses and ponies. Equine Veterinary Journal, v. 24, n. 1, p. 41-45, 1992.

ROBINSON, C. S.; TIMOFTE, D.; SINGER, E. R.; RIMMINGTON, L.; RUBIO-MARTINEZ, L. M. Prevalence and antimicrobial susceptibility of bacterial isolates from horses with synovial sepsis: A cross-sectional study of 95 cases. The Veterinary Journal, v. 216, p. 117-121, 2016.

SAMPIERI, F.; HINCHCLIFF, K. W.; TORIBIO, R. E. Tetracycline therapy of Lawsonia intracellularis enteropathy in foals. Equine Veterinary Journal, v. 38, n. 1, p. 89-92, 2006.

SANTSCHI, E. M.; PAPICH, M. G. Pharmacokinetics of gentamicin in mares in late pregnancy and early lactation. Journal of Veterinary Pharmacology and Therapeutics, v. 23, p. 359$363,2000$.

SAPADIN, A. N.; FLEISHMAJER, R. Tetracyclines: non antibiotic properties and their clinical implications. Journal of the American Academy of Dermatology, v. 54, n. 2, p. 258-265, 2006. 
SCHNABEL, L. V.; PAPICH, M. G.; WATTS, A. E.; et al. Orally administered doxycycline accumulates in synovial fluid compared to plasma. Equine Veterinary Journal, v. 42, n. 3, p. 208-212, 2010.

SPEER, B. S.; SHOEMAKER, N. B.; SALYERS, A. A. Bacterial resistance to tetracycline: mechanisms, transfer and clinical significance. Clinical Microbiology Reviews, v. 5, n. 4, p. 387-399, 1992.

WANG, N.; TIAN, X.; CHEN, Y.; et al. Low dose doxycycline decreases systemic inflammation and improves glycemic control, lipid profiles, and islet morphology and function in $\mathrm{db} / \mathrm{db}$ mice. Scientific Reports, v. 7, n. 1, 2017. doi: 10.1038/s41598-017-14408-7

WELLING, P. G.; KOCH, P. A.; LAU, C. C.; et al. Bioavailability of tetracycline and doxycycline in fasted and nonfasted subjects. Antimicrobial Agents and Chemotherapy, v. 11, n. 3, p. 462469, 1977.

WOMBLE, A.; GIGUÈRE, S.; LEE, E. A. Pharmacokinetics of oral doxycycline and concentrations in body fluids and bronchoalveolar cells of foals. Journal of Veterinary

Pharmacology Therapy, v. 30, p. 187-193, 2007.

Autor para correspondência: Bruna da Rosa Curcio. Hospital de Clínicas Veterinárias, Campus Universitário Capão do Leão, S/N, CEP 96160-000,

Capão do Leão (RS). curciobruna@hotmail.com 